\title{
The Relationship between Acute Functional Status and Long-Term Ambulation after Severe Traumatic Brain Injury
}

\author{
Elaine de Guise, ${ }^{1,2}$ Joanne LeBlanc, ${ }^{1}$ Michel Abouassaly, ${ }^{1}$ Howell Lin, ${ }^{1}$ \\ Julie Lamoureux, ${ }^{3}$ Marie-Claude Champoux, ${ }^{1}$ Céline Couturier, ${ }^{1}$ Mohammed Maleki, ${ }^{2}$ \\ Eric P. Roger, ${ }^{4}$ and Mitra Feyz ${ }^{1}$ \\ ${ }^{1}$ Traumatic Brain Injury Program, McGill University Health Centre (Montreal General Hospital), Montreal, QC, Canada H3G $1 A 4$ \\ ${ }^{2}$ Department of Neurology and Neurosurgery, McGill University, Montreal, QC, Canada H3A OG4 \\ ${ }^{3}$ Social and Preventive Medicine Department, University of Montreal, Montreal, QC, Canada H3C 3J7 \\ ${ }^{4}$ Department of Neurosurgery, University at Buffalo, The State University of New York, Buffalo, NY 14260-1660, USA
}

Correspondence should be addressed to Elaine de Guise, elaine.deguise@muhc.mcgill.ca

Received 1 May 2012; Accepted 29 May 2012

Academic Editors: M. Schmitter-Edgecombe, J. J. Sosnoff, and M. Syczewska

Copyright (C) 2012 Elaine de Guise et al. This is an open access article distributed under the Creative Commons Attribution License, which permits unrestricted use, distribution, and reproduction in any medium, provided the original work is properly cited.

\begin{abstract}
Objective. To correlate long-term physical impairments of patients with severe traumatic brain injury (sTBI) based on their functional status in an acute care setting. Methods. 46 patients with sTBI participated in this prospective study. The Extended Glasgow Outcome Scale (GOSE) and the FIM instrument were rated at discharge from the acute care setting and at followup. The Functional Ambulation Classification (FAC), the Five-Meter Gait Speed, a quantified measure of negotiating stairs (Stair Climbing Speed and Rails used), and the functional reach test were rated at followup. Results. The subject with a score of 6 on the GOSE at discharge remained nonfunctional ambulator at followup. None of the subjects with a GOSE score of 5 became independent ambulators. Fifty percent of the subjects with a GOSE score of 4 were dependent ambulators. 100\% of the subjects with a GOSE score of 2 or 3 at discharge were independent ambulators. A higher FIM score at discharge was associated with a greater chance of ambulating independently at 2 to 5 years after TBI $\left(\chi_{\mathrm{KW}^{2}}{ }_{2 d f}\right)$. Conclusions. These data will allow physical health professionals in acute rehabilitation settings to provide more precise long-term physical outcome information to patients and families.
\end{abstract}

\section{Introduction}

Traumatic brain injuries (TBI) are sudden and devastating and are a major cause of disability in adults [1]. The incidence of TBI is estimated to be over 1 million cases per year in North America [2-4], while the prevalence of TBI in Canada is close to 600 in 100,000 [2]. In Quebec alone, there were 11,000 cases of mild TBI in 2008 [3]. Annually in Canada, over 16,000 patients are hospitalized with a TBI [1] and of those, approximately 5,660 will sustain a severe TBI (sTBI). Survivors may be left with a combination of cognitive, behavioral, emotional, physical, and psychosocial difficulties that restrict participation in preinjury roles [5]. Many long-term outcome studies have concluded that patients with moderate to severe TBI show physical and functional improvement but remain with cognitive and psychosocial adjustment problems [6-11].

Several research papers have been published on the cognitive and psychosocial outcomes of TBI but very few have explored the physical outcome of these patients. Some longitudinal studies have revealed a significant motor function improvement while in rehabilitation $[12,13]$ with marked improvement between the first and sixth week after injury [14]. In a multicenter study [15] with patients from rehabilitation centres after sTBI, evaluation of paresis, ataxia, and postural instability showed a pattern of improvement over time with the greatest improvement occurring while the patient was in inpatient rehabilitation and at 6 month follow-up assessment. Moreover, the authors found the presence of a persistent involuntary movement disorder 
(tremor, chorea/athetosis, dystonia). Two years following rehabilitation, more than one-third of subjects continued to display a neuromotor abnormality on basic neurological examination. The most common deficit was the persistence of tandem gait abnormality [15]. A study done by Hillier and colleagues (1997) showed the presence of balance deficits as well as hemiparesis in 15 percent and quadriparesis in 6 percent of their patients with TBI 5 years after injury [16].

Several other studies on physical outcome following TBI focused on functional aspects using global measures of disability. Using the FIM instrument, a phone followup study revealed that most of the patients with mild to moderate TBI were physically independent at 3 and 6 months after TBI [17]. Another study showed that 73 percent of patients with sTBI were ambulatory at less than 6 months following the injury [18]. With regard to specific physical variables, only a few outcome studies have been done. One by Engender et al. (1996) [19] reported that TBI patients who present with moderate or severe incoordination on admission to rehabilitation were at higher risk of needing physical assistance for mobility and self-care at discharge and at one year followup. In addition, early residual motor impairment after sTBI during rehabilitation was associated with poorer vocational outcome one year after injury [20]. Other studies have demonstrated that balance deficits present on admission to rehabilitation predict worse outcome at discharge $[21,22]$.

To our knowledge, all long-term sTBI outcome studies looking at motor functioning involved subjects who were followed in rehabilitation [8-10, 23-26] and who were reevaluated at followup one year following trauma. No investigation has included patients with a more severe injury or a poorer outcome who never reached candidacy for rehabilitation and who needed to be placed in a longterm care facility. These studies present with a selection bias as not all survivors of sTBI were included. Longterm outcome of patients who benefited from rehabilitation following sTBI and those who did not probably differs. Investigation of long-term outcome for all survivors of sTBI irrespective of their discharge destination from acute care would allow a more complete picture of outcome in this patient population. Thus, further investigation in this area could help patients and their families as well as physical rehabilitation professionals of the acute care treating teams understand what variables predict long-term physical outcome in sTBI. Data in this area would allow them to be in a better position to answer the question often asked in the acute care setting "Will he/she be able to walk again?" In addition, a clearer picture of long-term physical outcome based on status in acute care could help improve the focus and quality of services by offering appropriate intervention early to this population.

The goal of this study is to explore the relationship between physical status of patients with sTBI while in the acute care setting and at followup 2 to 5 years after injury. Based on the results of previous research of these patients while in rehabilitation, there should be a positive relationship between outcome at discharge from acute care and physical status at followup.

\section{Method}

2.1. Participants. All survivors of sTBI (i.e., Glasgow Coma Score (GCS) $\leq 8$ after resuscitation) admitted to the McGill University Health Center (MUHC) adult neurotrauma program from April 1999 to March 2002, who had progressed through at least two years of recovery, were invited to attend a clinical followup for the sole purpose of this study. This investigation was approved by the Research Ethics Board of the MUHC. All charts were reviewed to ensure accuracy of the GCS score. Retrospective data were collected from the Trauma Registry and the TBI program data bank by experienced support staff members of the TBI program.

Data on a total of 171 patients with sTBI were collected. 46 patients participated in the study ( 83 died before discharge, seven died after discharge, 17 refused to participate, 15 were unreachable, and three were ineligible-deemed incompetent). Among this group of participants, 30 had received specialized TBI intensive rehabilitation following trauma, three were referred to a specialized TBI out-patient rehabilitation setting, and 13 were transferred to a long-term care facility (nursing home).

2.2. Descriptive Variables. The following descriptive variables were obtained from chart review for each patient: gender, age, educational level, and mechanism of injury. Other variables gathered at followup included marital status, living arrangements, and employment. A complete description of the sample has been published elsewhere [11].

2.3. Procedure. During their stay in acute care, experienced physiotherapists and occupational therapists rated physical and functional skills with the Extended Glasgow Outcome Scale (GOSE) [27] and the FIM instrument [28]. These measures were also administered at followup. At followup, the patients in the study carried out physical assessment tasks described below. On a total of 46 patients, twelve of them needed a wheelchair and were not able to actively and safely participate in the physical tasks.

Specific activities were designed to enable scoring of the physical FIM scale [28]. These activities began with mobilizing into the testing area, transferring/sitting into an armchair, and having a snack using a utensil. If ambulatory, the patient then stood and proceeded to an adapted bathroom 20-25 meters away. The next activities included performing a toilet transfer, brushing teeth and combing hair, putting on a hospital gown/shorts and a pair of slippers, transferring into the tub and simulating washing (wiping self with towel), and then walking up and down one flight of stairs. The patient or companion/caregiver was asked about personal toileting.

\subsection{Measures Administered at Discharge from Acute Care.} The Extended Glasgow Outcome Scale (GOSE) [27] was used to assess global outcome. The GOSE score, in this study, represented the interdisciplinary team's evaluation of patient outcome. On this scale, scores of 0 or 1 correspond to good recovery ( 0 = upper good recovery, $1=$ lower good recovery) 
referring to normal participation in social, vocational, and physical life. Scores of 2 or 3 indicate moderate disability ( 2 = upper moderate disability, 3 = lower moderate disability) describing the patient who is independent but physically or cognitively disabled and requiring an altered physical, social, psychological, or vocational environment for participation. Patients with severe disability receive scores of 4 (upper severe disability) or 5 (lower severe disability) and are totally dependent in managing a normal or modified environment, whereas a score of 6 corresponds to a vegetative state reflecting total dependency with no awareness of the environment. Patients who die received a score of 7.

The FIM instrument [28] was used to measure global functional levels [28]. It is an 18-item seven point scale, with increasing values indicating greater level of independence. The 18 items assess levels of self-care, continence, mobility, communication, and cognition. Three ratings were used in this study. The first was a global rating including the 18 items for a total ranging from a minimum of 18 to a maximum of 126. The second was a physical rating including those items related to activities of daily living, continence, and mobility with a range of 13 to 91 . The last rating included the cognitive domains consisting of social interaction, problem solving, memory, expression, and comprehension and ranged from 5 to 35 .

2.5. Measures Administered at Followup (2-5 Years). The Functional Ambulation Classification (FAC) [29] is a standardized test of independence in ambulation with quality of ambulation assessed using a scale from 1 (nonambulatory) to 6 (normal ambulation).

The Five-Metre Gait Speed [30] is a standardized test assessing speed (seconds) of ambulation for a distance of 5 meters. The distance was marked on the floor. Patients must ambulate unassisted but may be supervised and they were asked to walk uninterrupted at a comfortable speed. Timing starts when the first foot crosses the first marker and stops when the same leg crosses the second marker.

A quantified measure of negotiating stairs was included in the physical assessment. This included looking at speed of going up and down and whether or not rails were used. Time to climb up and down 10 steps was measured (Stair Climbing Speed). Subjects were asked to go up one flight of stairs and stop at the top before coming back down. The flight of stairs consisted of ten steps, each $18 \mathrm{~cm}$ high, $141 \mathrm{~cm}$ wide, $30 \mathrm{~cm}$ deep, and at an angle of inclination of $30^{\circ}$. Subjects were instructed to proceed at a comfortable speed and were neither encouraged nor discouraged from using the side rail, available on either side. Utilization of a railing during stair ambulation (rails used) was noted in a dichotomized fashion: yes (using the rails) or no (not using the rails).

Functional reach [31] is a standardized tool measuring forward maximal reach and balance. Patients, standing behind a line on the floor with arms at shoulder level, were asked to make a fist and extend their arm as far forward as they could without losing their balance or moving their feet. The position of the end of the third metacarpal was recorded $(\mathrm{cm})$. The task was repeated three times and the average distance reached was taken for the three trials. Although not a mobility measure, the functional reach test was used to quickly assess balance during the evaluation process.

2.6. Statistical Analyses. Descriptive statistics were carried out for basic subject description and for the various functional and physical measures. Means and standard deviations were computed for numerical variables and proportions were used for categorical variables. The relationship between functional and physical outcomes was assessed using Pearson's and Spearman's rank correlation coefficients. To compare various subgroups, a Kruskal-Wallis test was used to be conservative in light of the small subgroup sizes and the deviation from normality of the dependent variables. A level of significance of 0.05 was used for all those analyses. To determine how demographic variables influenced the association between early function and long-term ambulation, we ran stepwise regression models. In order to normalize the residuals, we had to transform the dependent variables using a natural logarithm. For the stepwise regressions, the specifications were $\mathrm{p}($ in $)<0.05$ and $\mathrm{p}($ out $)>0.06$. All analyses were done using SPSS 17.0.2 (PASW, IBM, Armonk, NY).

\section{Results}

3.1. Descriptive Statistics for Demographics at Baseline. The sample of 46 subjects was composed of 10 women (21.7\%) and 36 men $(78.3 \%)$. Age varied between 17 and 80 years old, with a mean and standard deviation of $40.68 \pm 17.28$. Five patients had one to six years of education (11\%), 29 $(66 \%)$ had seven to 13 years of education, and ten patients (23\%) had more than 14 years of education. Twenty-seven subjects (58.7\%) were working (23 full time and four part time) before the trauma and $11(23.9 \%)$ were not (seven unemployed and four retired). Eight of the 46 subjects $(17.4 \%)$ were students. Sixteen subjects $(34.8 \%)$ had a spouse (married or common law) and were living together and $27(58.7 \%)$ had no spouse (single, separated, divorced, or widowed). Only three subjects (6.5\%) had a partner but were not living together. The most common cause of TBI in this sample was by motor vehicle crash $(37.0 \%)$, followed closely by falls (28.3\%). Other accidents accounted for $15.2 \%$ of causes of trauma, criminal acts for $8.7 \%$, suicide attempts for $6.5 \%$, and work-related accidents for $4.3 \%$. Eleven subjects $(23.9 \%)$ had a past medical history that influenced premorbid function and $8.7 \%$ had a previous TBI. Only one subject $(2.2 \%)$ had a previous mobility limitation (walking with a cane/walker), and two subjects (4.4\%) had previous cognitive limitations (one minor and one severe). An extensive description of the sample is published elsewhere [8]. The functional ambulation classification was distributed as follows: 29 subjects $(63 \%)$ were ambulatory independent, $5(11 \%)$ were ambulatory independent on level surfaces only, 3 (6\%) were ambulatory dependent on supervision, 2 (4\%) were ambulatory dependent on physical assistance and $7(15 \%)$ were nonfunctional ambulators. It should be pointed out that the FAC was significantly associated with 
gender (significantly more male subjects were ambulatory independent than women, $83 \%$ versus $40 \%, \chi^{2}{ }_{4 d f}=14.007$, $P=0.007$ ) and with age (as age increased, independence decreased, $\left.\chi_{\mathrm{KW}^{2}}{ }_{4 d f}=9.721, P=0.045\right)$. FAC was not associated with GCS at time of trauma, mechanism of injury, previous living arrangements, or previous marital status.

Statistical analysis showed no difference in gender, in the initial GCS score or in the GOS-E and FIM instrument scores at discharge from the acute trauma center between the subjects who participated and those who refused to participate in this study.

3.2. Association between the Extended Glasgow Outcome Scale (GOSE) at Discharge and the Functional Ambulation Classification Scale (FAC) at Followup. The FAC classification was significantly associated with GOSE at discharge. Associations are provided in Table 1. The subject with GOSE 6 (vegetative state) at discharge remained nonfunctional ambulator at followup. None of the subjects with GOSE 5 (lower severe disability) became independent ambulators. Fifty percent of the subjects with GOSE 4 (upper severe disability) remained in the dependent ambulator categories. The participant who had prior mobility limitations was included in this group. $100 \%$ of the subjects with GOSE 2 (upper moderate disability) or GOSE 3 (lower moderate disability) at discharge were in the independent ambulator category at followup. These associations were statistically significant $\left(\chi_{16 d f}^{2}=35.042, P=0.004\right)$.

\subsection{Association between the FIM Instrument at Discharge} and the Functional Ambulation Classification Scale (FAC) at Followup. Results of the FAC were grouped into 3 general categories for the purpose of between group comparisons: nonfunctional ambulator ( score $=1$ ), dependent ambulator ( score $=2,3$, and 4), and independent ambulator (score = 5 and 6). Table 2 shows the distribution of FIM scores at discharge within these functional ambulation categories.

Figure 1 shows the tendency for functionally independent groups to have a higher FIM score at discharge. A KruskalWallis chi-square supported this tendency $\left(\chi_{\mathrm{KW}}{ }^{2}{ }_{2 d f}=17.988\right.$, $P<0.001)$. The same results were observed for the subscales of the FIM: $\chi_{\mathrm{KW}^{2}}{ }_{2 d f}=17.472, P<0.001$ for the physical portion and $\chi_{\mathrm{KW}^{2}}{ }_{2 d f}=14.657, P=0.001$ for the cognitive portion.

3.4. Association between Discharge FIM Ratings and Other Physical Tests. Table 3(a) shows the correlation coefficients between the three discharge FIM scores and the four other functional tests, whereas Table 3(b) gives the partial correlation coefficients controlling for age. The correlation between FIM ratings at discharge was not significantly associated with gait speed at followup. The FIM cognitive rating at discharge was not significantly associated with any of the functional measures at followup. The FIM total score and the FIM physical score at discharge were both moderately associated with the Stairs Climbing tests at followup. Even after controlling for age, the FIM total score and the FIM physical score at discharge were both associated with the Stairs Climbing tests at followup.

We performed stepwise linear regressions on a natural logarithm transformation of the four above-mentioned physical tests. The predictive variables submitted to the stepwise regression were age at the time of trauma, gender, and FIM (components and total score) at discharge. For the 5 -meter gait speed, only gender was a significant predictor ( $b=0.723, t=3.413, P=0.002$ ) showing an increase in the speed for males compared to females.

For the stairs-climb speed up variable, gender was a significant predictor $(b=-0.842, t=3.584, P=0.001)$ as well as the FIM total score at discharge $(b=-.011, t=3.662$, $P=0.001)$. This can be interpreted as, within each gender, the FIM total score at discharge increases, the score for the stairs-climb speed up test will decrease. Similarly, keeping FIM total score at discharge equal, men will have a lower score than females on this stairs-climb speed up test. Both variables explained $46 \%$ of the variation in the stairs-climb speed up variable.

Similarly for the stairs-climb speed down variable, gender was a significant predictor $(b=-1.114, t=4.495$, $P<0.001)$ as well as the FIM total score at discharge $(b=$ $-.011, t=3.199, P=0.003)$. This can be interpreted as, within each gender, the FIM total score at discharge increases, the score for the stairs-climb speed down test will decrease. Similarly, keeping FIM total score at discharge equal, men will have a lower score than females on this stairs-climb down test. Both variables explained $50 \%$ of the variation in the stairs-climb speed up variable.

Finally for the functional reach variable, gender was a significant predictor $(b=0.428, t=3.681, P=0.001)$ as well as the FIM cognitive score at discharge $(b=.020, t=$ 3.098, $P=0.004)$. This can be interpreted as, within each gender, FIM the cognitive score at discharge increases, the score for the functional reach test will increase. Similarly, keeping FIM cognitive score at discharge equal, men will have a higher score than females on this functional reach test. Both variables explained $41 \%$ of the variation in the functional reach variable.

\section{Discussion}

This study aimed to better understand physical outcome of patients with sTBI by looking at the relationship between functional level at discharge from acute care and physical status at followup. At two to five years after, sTBI, 74\% of our cohort of patients was considered as independent ambulators, $11 \%$ as dependent ambulators, and $15 \%$ as nonambulators which is quite similar to what has been observed earlier in the recovery process with patients from rehabilitation centres [18] and in keeping with results of previously long-term outcome studies already published.

4.1. Relationships between Outcome at Discharge from Acute Care and Quality of Ambulation at Followup. The hypotheses of this investigation were accurate. The results findings mirror what was found with patients in rehabilitation 
TABLE 1: Distribution of GOSE at discharge and FAC at followup $(n=46)$.

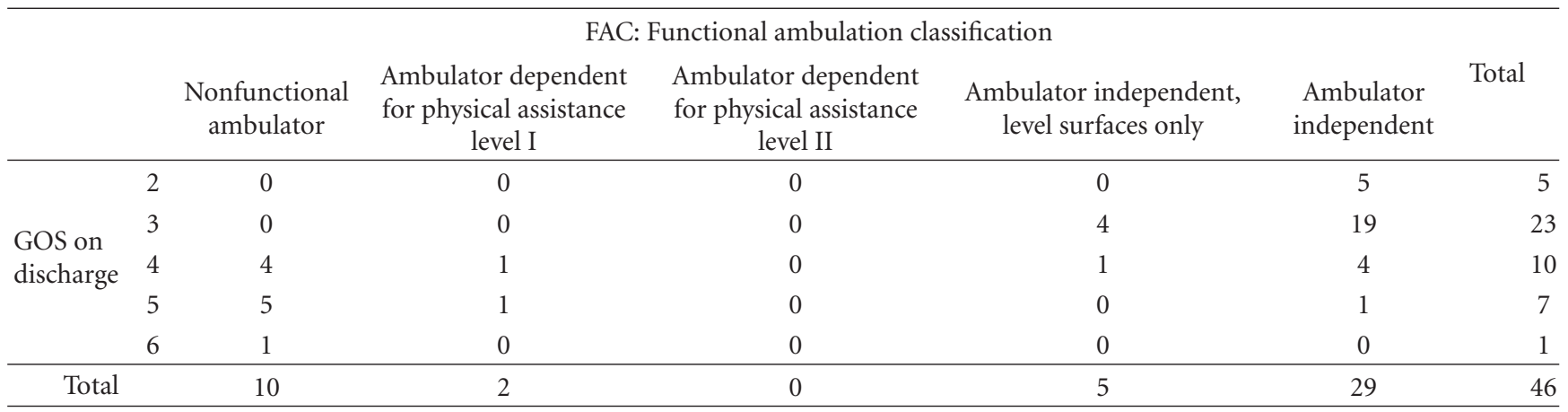

TABLE 2: Descriptive statistics for FIM scores at discharge in 3 ambulation groups $(n=46)$.

\begin{tabular}{|c|c|c|c|}
\hline Recoded ambulation & FIM total & FIM physical & FIM cognitive \\
\hline \multicolumn{4}{|c|}{ Nonfunctional ambulator $(n=7)$} \\
\hline Mean & 30.14 & 17.86 & 12.29 \\
\hline Median & 28.00 & 13.00 & 11.00 \\
\hline Variance & 96.810 & 63.810 & 28.905 \\
\hline Standard Deviation & 9.839 & 7.988 & 5.376 \\
\hline Minimum & 18 & 13 & 5 \\
\hline Maximum & 44 & 33 & 21 \\
\hline \multicolumn{4}{|c|}{ Ambulator dependent $(n=5)$} \\
\hline Mean & 49.00 & 37.60 & 11.40 \\
\hline Median & 50.00 & 39.00 & 13.00 \\
\hline Variance & 790.000 & 687.800 & 14.300 \\
\hline Standard Deviation & 28.107 & 26.226 & 3.782 \\
\hline Minimum & 18 & 13 & 5 \\
\hline Maximum & 92 & 79 & 14 \\
\hline \multicolumn{4}{|c|}{ Ambulator independent $(n=34)$} \\
\hline Mean & 81.41 & 60.53 & 20.88 \\
\hline Median & 85.50 & 60.00 & 20.00 \\
\hline Variance & 582.189 & 462.923 & 40.531 \\
\hline Standard Deviation & 24.129 & 27.516 & 6.366 \\
\hline Minimum & 21 & 15 & 6 \\
\hline Maximum & 123 & 91 & 34 \\
\hline
\end{tabular}

settings $[17-19,21,22]$ and show that functional abilities at the acute care level are positively correlated with ambulation quality at the community reintegration phase for all patients with TBI including the more severely impaired and regardless of whether they have been through a rehabilitation program. Moreover, age and gender were associated with the longterm quality of ambulation. Male and younger patients have a better level of ambulation few years after severe TBI. From a clinical point of view, patients with sTBI who have a better level of acute recovery and a better level of participation in social and physical activities in the acute environment (walking, transferring/sitting, eating, brushing teeth and combing hair, bathing, toileting, communicating, interacting with others, recalling information, behaving appropriately, etc.) have a greater chance of achieving a higher quality of ambulation 2 to 5 years following accident. The likelihood of walking without manual contact from another person or without need of standby guarding or verbal cueing is greater for patients with higher functioning at acute discharge. In addition, variability was greater for the FIM scores in individuals who became independent ambulators compared to subjects in the other classification groups. This variability might be explained by other variables, not controlled in this study, affecting return to ambulation.

4.2. Relationships between Outcome at Discharge and Physical Status at Followup. Similar reasoning would lead to the conclusion that patients who are at a higher functional level at acute discharge have a greater chance of being able to walk faster over a short distance as well as to climb stairs independently several years after sTBI. A surprising result concerned the relationship between the FIM cognitive score 


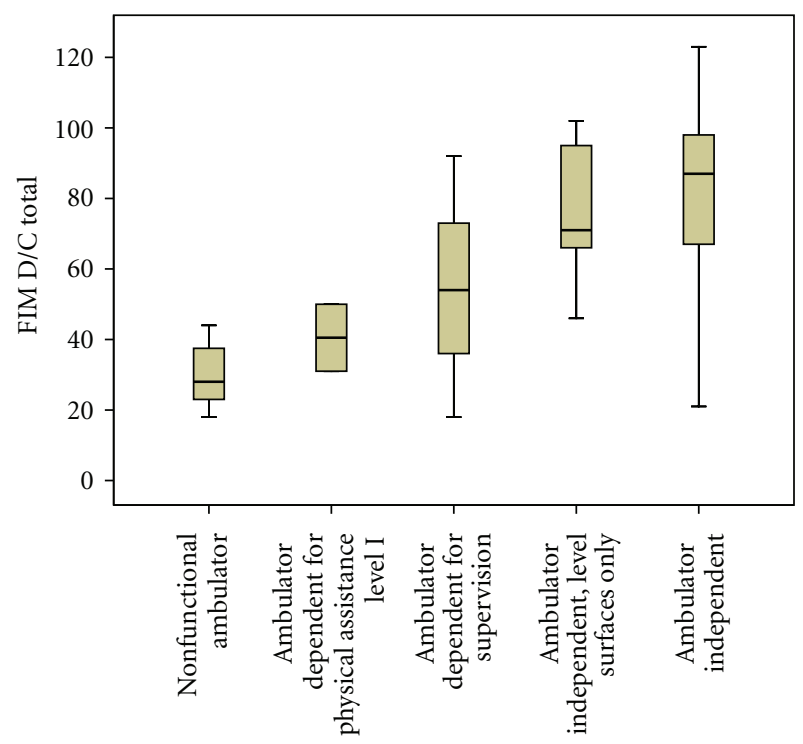

Functional ambulation classification

Figure 1: FIM score at discharge for ambulation categories $(n=$ 46).

and functional reach. It would seem that patients who have better memory, expression, comprehension, social interaction, and more appropriate behaviors are more likely to have better balance 2 to 5 years following TBI. Some studies have demonstrated the relationship between cognition and motor functions [32, 33]. A study done by Sosnoff et al. [32] showed a positive correlation between cognition and postural control tests in patients with mild traumatic brain injury (mTBI). They suggest that neural insults resulting in TBI lead to increased cognitive-motor association. They speculated that shared neural processes, such as visuospatial attention, is affected resulting in deficits in both cognitive and motor functions. More research on this specific topic is needed to verify these findings using different confounding variables that might influence the relationship between the FIM cognitive score and balance.

Correlations between FIM scores at discharge from acute care and gait speed and balance characteristics 2 to 5 years after injury in sTBI were not significant. Thus, association between the presence or not of gait speed and balance impairments and the level of physical functioning of patients with sTBI in the acute care setting cannot be made at this point in time. This absence of significance could be explained by a ceiling effect of the values obtained at followup. Other studies using more sensitive tests would therefore be required to obtain a significant relationship between acute physical outcome and gait speed and balance status following sTBI. It is also plausible that the relationship between physical outcome at this length of time after injury ( 2 to 5 years) with the acute physical outcome is not as great as at one year followup when most previous studies that showed significant physical predictive results were carried out [19-22].

4.3. Clinical Impact. In an acute care setting, sTBI often causes a catastrophic reaction for family and loved ones and they require a great deal of support. One way of providing support is to educate them on what is a TBI and the consequences of this injury. Very early after the accident, one of the first questions frequently asked is "Will he/she be able to walk again?" Based on the results of this study, more specific information to answer this question is now available. Patients in a vegetative state (GOSE $=6$ ) or who are at the lower severe disability level $($ GOSE $=5)$ at the end of their acute care stay have a lesser chance of ambulating independently on a long-term basis. Those who have upper or lower moderate disability at the end of their acute care stay (GOSE $=2-3$ ) have a better chance of becoming independent ambulators. The outcome for those patients who are at the upper moderate disability rating $($ GOSE $=4)$ is not as clear with $50 \%$ of them becoming independent ambulators in this study. More research is needed for this group to be able to obtain a clearer relationship between outcome at discharge from acute care and long-term functioning.

\section{Limitations and Conclusions}

One limitation of this study is the small sample size particularly for the dependent ambulator and the non-ambulatory groups. Only 46 of 171 eligible subjects participated in this investigation. Although those not included were statistically similar to the subjects studied (e.g., age, discharge FIM rating, discharge extended Glasgow Outcome Score) [11], there still exists some subject selection bias as only those who willingly came out for the study could be included. Moreover, because of the small sample, no analyses of regression were performed. Thus, statistically more powerful predictive statistics were not possible and only correlations between the variables were studied. Further investigation carried out with several acute care centres would allow recruitment of a greater number of patients.

In conclusion, $74 \%$ of patients with sTBI were able to ambulate independently 2 to 5 years after accident. In answer to the question posed by patients and families in the acute care setting regarding eventual ability to mobilize, healthcare professionals could therefore state that almost three quarters of patients following sTBI are eventually able to walk. The long-term prediction of gait speed or balance problems based on the functional status of patients in the acute care setting is not yet clear and future research is needed to better understand and predict physical outcome of patients with TBI.

\section{Disclosure}

The FIM data set, measurement scale, and impairment codes referenced herein are the property of Uniform Data System for Medical Rehabilitation, a division of UB Foundation Activities, Inc. The service marks and trademarks associated with the FIM instrument are all owned by Uniform Data System for Medical Rehabilitation, a division of UB Foundation Activities, Inc. 
TABLE 3

(a) Spearman correlation coefficients between the FIM ratings at discharge and other functional tests

\begin{tabular}{|c|c|c|c|}
\hline & D/C FIM total & D/C FIM physical score & D/C FIM cognitive score \\
\hline \multicolumn{4}{|l|}{ Five-meter gait $\mathrm{m} / \mathrm{sec}$} \\
\hline Spearman's rho & .158 & .186 & .016 \\
\hline Significant (2 tailed) & .374 & .326 & .931 \\
\hline$N$ & 34 & 34 & 34 \\
\hline \multicolumn{4}{|l|}{ Stair-climb speed up } \\
\hline Spearman's rho & $-.459^{* *}$ & $-.481^{* *}$ & -.221 \\
\hline Significant (2 tailed) & .006 & .004 & .208 \\
\hline$N$ & 34 & 34 & 34 \\
\hline \multicolumn{4}{|l|}{ Stair-climb down } \\
\hline Spearman's rho & $-.416^{*}$ & $-.446^{* *}$ & -.234 \\
\hline Significant (2 tailed) & .014 & .008 & .183 \\
\hline$N$ & 34 & 34 & 34 \\
\hline \multicolumn{4}{|l|}{ Functional reach (mean) } \\
\hline Spearman's rho & .164 & .109 & .333 \\
\hline Significant (2 tailed) & .355 & .539 & .055 \\
\hline$N$ & 34 & 34 & 34 \\
\hline
\end{tabular}

(b) Partial correlation coefficients between the FIM ratings at discharge and other functional tests controlling for age

\begin{tabular}{|c|c|c|c|}
\hline & D/C FIM total & D/C FIM physical score & D/C FIM cognitive score \\
\hline \multicolumn{4}{|l|}{ Five-meter gait $\mathrm{m} / \mathrm{sec}$} \\
\hline Pearson & .100 & .182 & -.312 \\
\hline Significant (2 tailed) & .594 & .326 & .088 \\
\hline$N$ & 29 & 29 & 29 \\
\hline \multicolumn{4}{|l|}{ Stair-climb speed up } \\
\hline Pearson & $-.547^{* *}$ & $-.504^{* *}$ & $-.382^{*}$ \\
\hline Significant (2 tailed) & .001 & .004 & .034 \\
\hline$N$ & 29 & 29 & 29 \\
\hline \multicolumn{4}{|l|}{ Stair-climb down } \\
\hline Pearson & $-.496^{* *}$ & $-.451^{* *}$ & $-.372^{*}$ \\
\hline Significant (2 tailed) & .005 & .011 & .039 \\
\hline$N$ & 29 & 29 & 29 \\
\hline \multicolumn{4}{|l|}{ Functional reach (mean) } \\
\hline Pearson & .134 & .066 & .273 \\
\hline Significant (2 tailed) & .473 & .723 & .137 \\
\hline$N$ & 29 & 29 & 29 \\
\hline
\end{tabular}

${ }^{*} P<0.05,{ }^{* *} P<0.01$.

\section{Conflict of Interests}

The authors report no conflict of interests.

\section{Acknowledgments}

This study was supported by grants from the McGill University Health Centre (Montreal General hospital Foundation) and by the Quebec Ministry of Health and Social Services.

\section{References}

[1] J. Cassidy, L. J. Carroll, J. Borg et al., "Incidence, risk factors and prevention of mild traumatic brain injury: result of the WHO collaborating centre task force on mild traumatic brain injury," Journal of Rehabilitation Medicine, vol. 43, pp. 28-60, 2004.

[2] Agence de la santé et des services sociaux de Montréal, "Projet d'organisation des services à l'intention des Montréalais ayant subi un traumatisme cranio-cérébral," 2011. 
[3] G. Iverson, "Mild traumatic brain injury meta-analyses can obscure individual differences," Brain Injury, vol. 24, no. 10, pp. 1246-1255, 2010.

[4] Canadian Institute for Health Information (CIHI), "Hospitalizations due to traumatic brain injuries down 35\% over a decade. CIHI Web site," August 2006, https://secure.cihi.ca/ free_products/ntr_head_injuries_2006_f.pdf.

[5] T. Hart, J. Whyte, M. Polansky et al., "Concordance of patient and family report of neurobehavioral symptoms at 1 year after traumatic brain injury," Archives of Physical Medicine and Rehabilitation, vol. 84, no. 2, pp. 204-213, 2003.

[6] S. S. Dikmen, J. E. Machamer, J. M. Powell, and R. Temkin, "Outcome 3 to 5 years after moderate to severe traumatic brain injury," Archives of Physical Medicine and Rehabilitation, vol. 84, no. 10, pp. 1449-1457, 2003.

[7] D. Hoofien, A. Gilboa, E. Vakil, and P. J. Donovick, “Traumatic brain injury (TBI) 10-20 years later: a comprehensive outcome study of psychiatric symptomatology, cognitive abilities and psychosocial functioning," Brain Injury, vol. 15, no. 3, pp. 189209, 2001.

[8] A. Colantonio, G. Ratcliff, S. Chase, S. Kelsey, M. Escobar, and L. Vernich, "Long term outcomes after moderate to severe traumatic brain injury," Disability and Rehabilitation, vol. 26, no. 5, pp. 253-261, 2004.

[9] G. Ratcliff, A. Colantonio, M. Escobar, S. Chase, and L. Vernich, "Long-term survival following traumatic brain injury," Disability and Rehabilitation, vol. 27, no. 6, pp. 305-314, 2005.

[10] R. L. Wood and N. A. Rutterford, "Psychosocial adjustment 17 years after severe brain injury," Journal of Neurology, Neurosurgery and Psychiatry, vol. 77, no. 1, pp. 71-73, 2006.

[11] E. de Guise, J. LeBlanc, M. Feyz et al., "Long-term outcome after severe traumatic brain injury: The McGill Multidisciplinary Prospective Outcome Study," Journal of Head Trauma Rehabilitation, vol. 23, no. 5, pp. 294-303, 2008.

[12] R. K. Bode and A. W. Heinemann, "Course of functional improvement after stroke, spinal cord injury, and traumatic brain injury," Archives of Physical Medicine and Rehabilitation, vol. 83, no. 1, pp. 100-106, 2002.

[13] L. Cook, D. S. Smith, and G. Truman, "Using functional independence measure profiles as an index of outcome in the rehabilitation of brain-injured patients," Archives of Physical Medicine and Rehabilitation, vol. 75, no. 4, pp. 390-393, 1994.

[14] B. Swaine and S. L. Sullivan, "Longitudinal profile of early motor recovery following severe traumatic brain injury," Brain Injury, vol. 10, no. 5, pp. 347-366, 1996.

[15] W. C. Walker and T. C. Pickett, "Motor impairment after severe traumatic brain injury: a longitudinal multicenter study," Journal of Rehabilitation Research and Development, vol. 44, no. 7, pp. 975-982, 2007.

[16] S. L. Hillier, M. H. Sharpe, and J. Metzer, "Outcomes 5 years post-traumatic brain injury (with further reference to neurophysical impairment and disability)," Brain Injury, vol. 11, no. 9, pp. 661-675, 1997.

[17] M. L. Dombovy and A. C. Olek, "Recovery and rehabilitation following traumatic brain injury," Brain Injury, vol. 11, no. 5, pp. 305-318, 1997.

[18] D. I. Katz, D. K. White, M. P. Alexander, and R. B. Klein, "Recovery of ambulation after traumatic brain injury," Archives of Physical Medicine and Rehabilitation, vol. 85, no. 6, pp. 865-869, 2004.

[19] J. S. Engender, D. X. Cifu, J. Wright et al., "The impact of acute complications, fractures, and motor deficits on functional outcome and length of stay after traumatic brain injury: a multicenter analysis," Journal of Head Trauma Rehabilitation, vol. 11, no. 5, pp. 15-26, 1996.

[20] D. X. Cifu, L. Keyser-Marcus, E. Lopez et al., "Acute predictors of successful return to work 1 year after traumatic brain injury: a multicenter analysis," Archives of Physical Medicine and Rehabilitation, vol. 78, no. 2, pp. 125-131, 1997.

[21] K. Black, R. Zafonte, S. Millis et al., "Sitting balance following brain injury: does it predict outcome?" Brain Injury, vol. 14, no. 2, pp. 141-152, 2000.

[22] G. Juneja, J. J. Czyrny, and R. T. Linn, "Admission balance and outcomes of patients admitted for acute inpatient rehabilitation," American Journal of Physical Medicine and Rehabilitation, vol. 77, no. 5, pp. 388-393, 1998.

[23] L. Mailhan, P. Azouvi, and A. Dazord, "Life satisfaction and disability after severe traumatic brain injury," Brain Injury, vol. 19, no. 4, pp. 227-238, 2005.

[24] F. M. Hammond, K. D. Grattan, H. Sasser et al., "Five years after traumatic brain injury: a study of individual outcomes and predictors of change in function," Neurorehabilitation, vol. 19, no. 1, pp. 25-35, 2004.

[25] J. T. King, P. M. Carlier, and D. W. Marion, "Early Glasgow outcome scale scores predict long-term functional outcome in patients with severe traumatic brain injury," Journal of Neurotrauma, vol. 22, no. 9, pp. 947-954, 2005.

[26] C. O’Connor, A. Colantonio, and H. Polatajko, “Long term symptoms and limitations of activity of people with traumatic brain injury: a ten-year follow-up," Psychological Reports, vol. 97, no. 1, pp. 169-179, 2005.

[27] B. Jennett, J. Snoek, M. R. Bond, and N. Brooks, "Disability after severe head injury: observations on the use of the Glasgow outcome scale," Journal of Neurology Neurosurgery and Psychiatry, vol. 44, no. 4, pp. 285-293, 1981.

[28] C. V. Granger, A. C. Cotter, B. B. Hamilton, and R. C. Fiedler, "Functional assessment scales: a study of persons with multiple sclerosis," Archives of Physical Medicine and Rehabilitation, vol. 71, no. 11, pp. 870-875, 1990.

[29] M. K. Holden, K. M. Gill, and M. R. Magliozzi, "Gait assessment for neurologically impaired patients. Standards for outcome assessment," Physical Therapy, vol. 66, no. 10, pp. 15301539, 1986.

[30] T. Oberg, A. Karsznia, and K. Oberg, "Basic gait parameters: reference data for normal subjects, 10-79 years of age," Journal of Rehabilitation Research and Development, vol. 30, no. 2, pp. 210-223, 1993.

[31] P. W. Duncan, D. K. Weiner, J. Chandler, and W. Poewe, "Functional reach: a new clinical measure of balance," Journal of Gerontology and Medicine Sciences, vol. 45, no. 6, pp. M192M197, 1990.

[32] J. J. Sosnoff, S. P. Broglio, and M. S. Ferrara, "Cognitive and motor function are associated following mild traumatic brain injury," Experimental Brain Research, vol. 187, no. 4, pp. 563571, 2008.

[33] J. Konczak and D. Timmann, "The effect of damage to the cerebellum on sensorimotor and cognitive function in children and adolescents," Neuroscience and Biobehavioral Reviews, vol. 31, no. 8, pp. 1101-1113, 2007. 


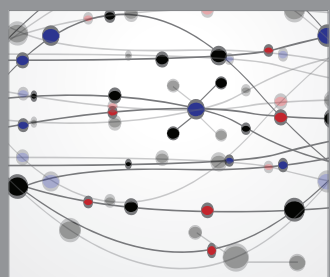

The Scientific World Journal
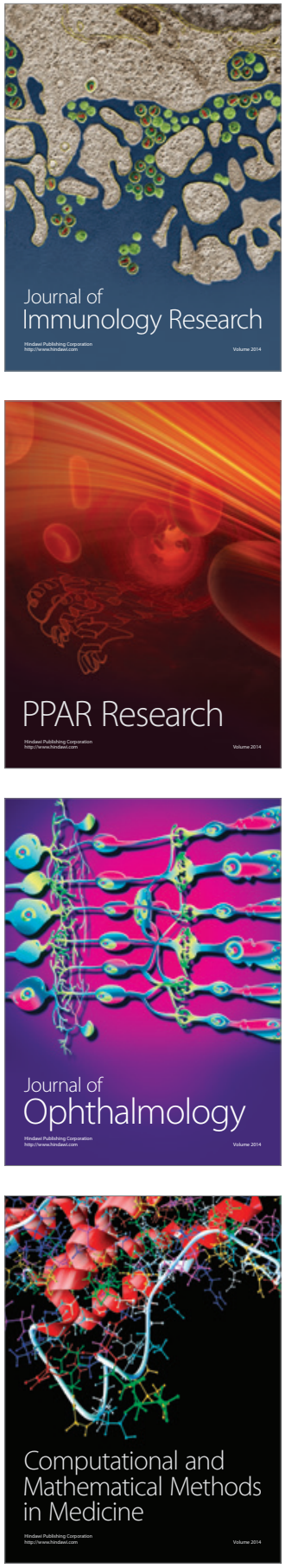

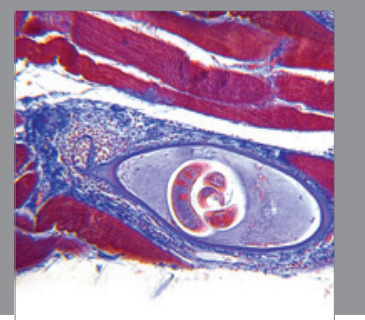

Gastroenterology

Research and Practice
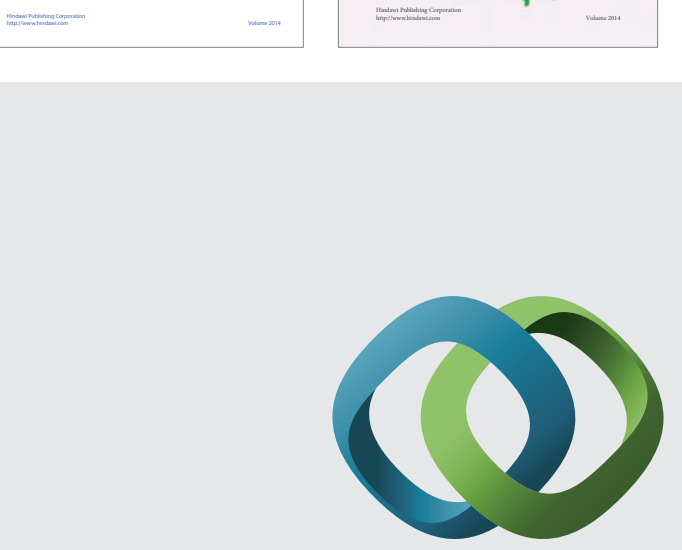

\section{Hindawi}

Submit your manuscripts at

http://www.hindawi.com
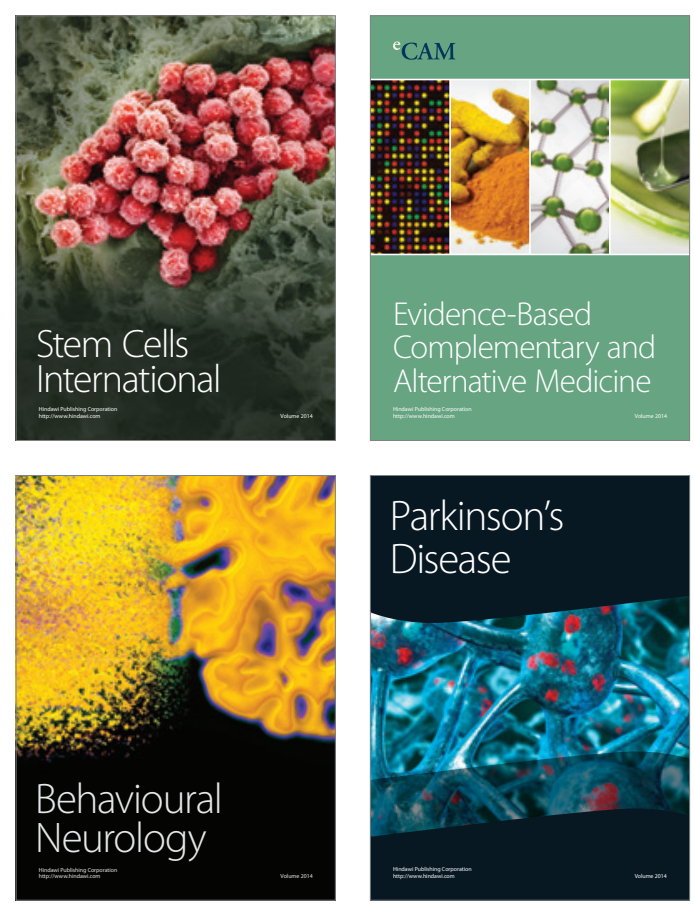

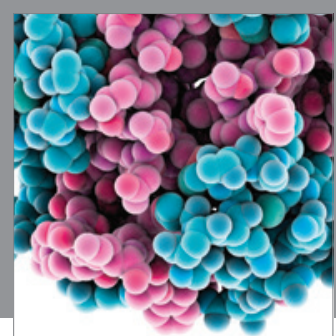

Journal of
Diabetes Research

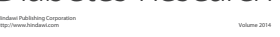

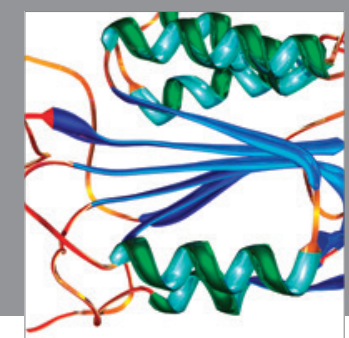

Disease Markers
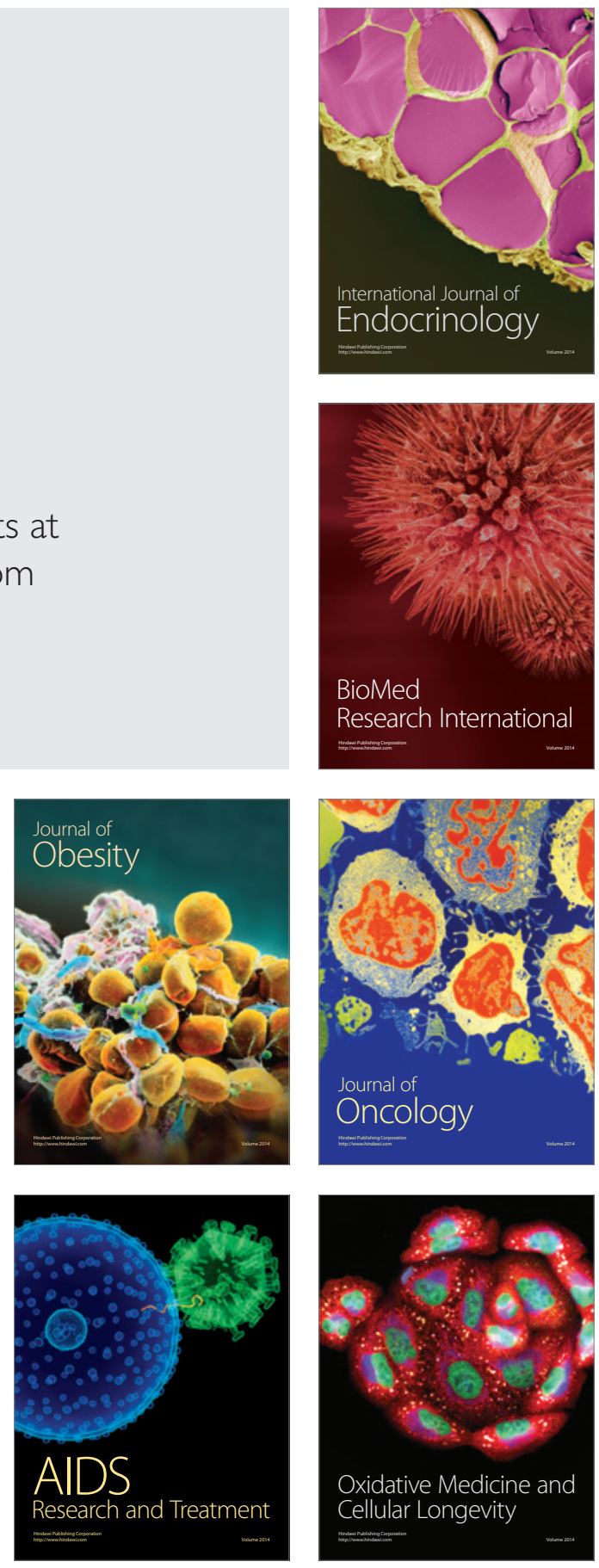